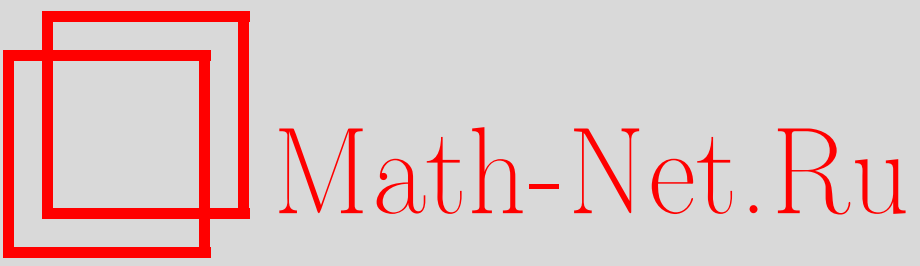

В. А. Артамонов, Действия точечных алгебр Хопфа на квантовых многочленах, УМH, 2000, том 55, выпуск 6, 125-126

DOI: https://doi.org/10.4213/rm337

Использование Общероссийского математического портала Math-Net.Ru подразумевает, что вы прочитали и согласны с пользовательским соглашением

http://www.mathnet.ru/rus/agreement

Параметры загрузки:

IP: 34.229 .45 .116

26 апреля 2023 г., 16:58:37 


\title{
ДЕЙСТВИЯ ТОЧЕЧНЫХ АЛГЕБР ХОПФА НА КВАНТОВЫХ МНОГОЧЛЕНАХ
}

\author{
В. А. Артамонов
}

Пусть $k$-поле, в котором фиксирована квадратная матрица $Q=\left(q_{i j}\right)$ размера $n \geqslant 3$, причем элементы $q_{i j} \in k^{*}, i, j=1, \ldots, n$, удовлетворяют условию $q_{i i}=q_{i j} q_{j i}=1$ для всех $i, j$.

ОпредЕлЕниЕ. Через $\Lambda=k_{Q}\left[X_{1}, \ldots, X_{n}\right]$ обозначается ассоциативная $k$-алгебра с единицей, порожденная элементами $X_{1}, \ldots, X_{n}$ с определяюшими соотношениями $X_{i} X_{j}=q_{i j} X_{j} X_{i}$, $1 \leqslant i, j \leqslant n$. Алгебра $\Lambda$ называется алгеброй квантовых многочленов. Элементы $q_{i j}$ назьваются мультипараметрами.

Алгебра $\Lambda$ удовлетворяет условию Оре и потому имеет тела частных $F=k_{Q}\left(X_{1}, \ldots, X_{n}\right)$.

ОПредЕЛЕнИЕ. Алгебра $\Lambda$ назьвается алгеброй общих квантовых многочленов, если все мультипараметры $q_{i j}, 1 \leqslant i<j \leqslant n$, независимы в мультипликативной абелевой группе $k^{*}$ поля $k$. Другими словами, если $m_{i j} \in \mathbb{Z}$, то

$$
\prod_{1 \leqslant i<j \leqslant n} q_{i j}^{m_{i j}}=1 \Leftrightarrow m_{i j}=0
$$

Всюду в дальнейшем предполагается, что $\Lambda$ - алгебра общих квантовых многочленов. В работе [1] рассмотрены автоморфизмы алгебры $\Lambda$ и их инварианты.

ТеОРема 1 [1]. Пусть $\gamma-$ инвективный әндоморфизм алгебры $\Lambda$. Тогда существуют такие әлементы $\gamma_{1}, \ldots, \gamma_{n} \in k^{*}$, что $\gamma\left(X_{j}\right)=\gamma_{j} X_{j}, j=1, \ldots, n$. В частности, $\gamma$ является автоморфизмам и группа Aut $\Lambda$ абелева.

Tеорема 2 [1]. Пусть $G$ - конечная подгруппа в Aut $\Lambda$. Тогда подалгебра инвариантов $\Lambda^{G}$ есть нетерова слева и справа алгебра, причем $\Lambda$ - конечнопорожденный левый и правый $\Lambda^{G}$-модуль. Действие $G$ продолжается на тело частных $F$, причем подтело инвариантов $F^{G}$ является телом частных подалгебры $\Lambda^{G}$.

Рассмотрим обобщения этих результатов, связанные с изучением (ко)действий алгебр Хопфа на алгебрах общих квантовых многочленов $\Lambda$. Напомним, что для произвольной алгебры Хопфа $H$ через $P(H)$ обозначается (ограниченная) алгебра Ли примитивных элементов, а через $G(H)$ - группа всех элементов из $H$.

ТЕорема 3. Пусть задана коммутативная полупростая алгебра Хопфа $H$, для которой $\Lambda$ является $H$-комодульной алгеброй со структурным морфизмом $\rho: \Lambda \rightarrow H \otimes_{k} \Lambda$. Тогда существуют такие әлементы $\alpha_{1}, \ldots, \alpha_{n} \in G(H)$, что $\rho\left(X_{i}\right)=\alpha_{i} \otimes X_{i}$ для всех $i=1, \ldots, n$.

Для дальнейшего нам потребуется описание алгебры Ли $\operatorname{Der} \Lambda$ дифференцирований $\Lambda$. Подалгебра Derint $\Lambda$ внутренних дифференцирований является идеалом в Der $\Lambda$. Кроме того, имеются дифференцирования $\partial_{1}, \ldots, \partial_{n}$, где $\partial_{j}\left(X_{i}\right)=\delta_{i j} X_{i}$. Отметим, что $\left[\partial_{i}, \partial_{j}\right]=0,\left[\partial_{i}, \operatorname{ad}_{u}\right]=$ $\operatorname{ad}_{\partial_{i} u}$. Кроме того, если char $k=p>0$, то $\partial_{i}^{p}=\partial_{i}$. Таким образом, линейная оболочка $L$ дифоференцирований $\partial_{1}, \ldots, \partial_{n}$ является абелевой алгеброй Ли размерности $n$. Следующая теорема выводится из работы [2].

ТеОРема 4. Имеется прямое разложение векторных пространств $\operatorname{Der} \Lambda=$ Derint $\Lambda \oplus L$. Любая конечномерная подалгебра Ли в Derint $\Lambda$ является абелевой.

Работа выполнена при частичной поддержке Российского фонда фундаментальных исследований (гранты №№ 99-01-00382, 00-15-96128) и программы “Университеты России” № 5527. 
ПреДЛОЖениЕ 5. Пусть $\operatorname{char} k=0$ и $\partial$ - произвольное дифференцирование $\Lambda$. Предполохим, что существует такой ненулевой многочлен $f(T) \in k[T]$, что $f(\partial)=0$. Тогда $\partial=0$.

Пусть $U$ - универсальная (ограниченная) обертывающая алгебра для Der. Группа автоморфизмов $G=$ Aut $\Lambda$ действует на $U$ с помошью сопряжений. Действительно, по теореме 1

$$
\alpha \partial_{j} \alpha^{-1}=\partial_{j}, \quad \alpha \operatorname{ad}_{u} \alpha^{-1}=\operatorname{ad}_{\alpha(u)} .
$$

Положим $H_{\Lambda}=U \sharp k G$. Тогда $\Lambda$ является $H_{\Lambda}$-модульной алгеброй.

Теорема 6. Пусть поле $k$ алгебраически замкнуто и имеет нулевую характеристику. Предположим, что $H$ - кокоммутативная алгебра Хопфа, для которой $\Lambda$ является $H$-модульной алгеброй. Тогда существуют такой гомоморфизм алгебр Хопфа $\xi: H \rightarrow H_{\Lambda}$, что действие $H$ в $\Lambda$ индуцировано морфизмом $\xi$ и действием $H_{\Lambda}$ на $\Lambda$.

ОпредЕлЕниЕ [3; с. 45]. Пусть $H$ - алгебра Хопфа, действующая на $\Lambda$. Расширение $\Lambda / \Lambda^{H}$ называется целым в смысле Шелтера, если для любого элемента $f \in \Lambda$ найдется такое натуральное число $m$, что $f^{m}+q(f)=0$, где $q$ - сумма элементов вида

$$
a_{1} f^{n_{1}} \cdots a_{d} f^{n_{d}} a_{d+1}
$$

для некоторых $a_{1}, \ldots, a_{d+1} \in \Lambda^{H}$, где $n_{1}+\cdots+n_{d}<m$.

ПРеДЛОЖЕнИЕ 7. Пусть $H$ - подалгебра Хопфа в $H_{\Lambda}$ и поле $k$ алгебраически замкнуто и имеет нулевую характеристику. Предположим, что расширение $\Lambda / \Lambda^{H}$ является целым в смысле Шелтера. Если $P(H) \neq 0$, то $\Lambda^{H}$ и $P(H)$ коммутативны.

Теорема 8. Пусть $H$ - подалгебра Хопфа в $H_{\Lambda}$. Действие $H$ на $\Lambda$ можно продолжить на тело частных $F=k_{Q}\left(X_{1}, \ldots, X_{n}\right)$. Предположим, что $F$ имеет конечную левую (правую) размерность над подтелом инвариантов $F^{H}$. Пусть характеристика поля $k$ равна нулю и некоторый мультипараметр $q_{i j}, 1 \leqslant i<j \leqslant n$, алгебраччески независим над полем рациональных чисел $\mathbb{Q} \subseteq k$. Тогда $P(H)=0$, и поэтому $H=k G$, əде $G-$ noдəpynna в Aut $\Lambda$.

ОпРЕДЕЛЕнИЕ. Алгебра Хопфа $H$ над полем $k$ называется точечной, если все простые коподалгебры в $H$ имеют размерность 1.

ТЕОрема 9. Пусть $H$ - точечная конечномерная алгебра Хопфа, действующая на алгебре общих квантовых многочленов $\Lambda, n \geqslant 3$. Предположим, что поле $k$ содержит примитивный корень из 1 степени $\operatorname{dim} H u \operatorname{char} k$ взаимно проста $c \operatorname{dim} H$. Пусть $I-$ аннулятор $\Lambda$ в $H$. Тогда I является идеалом Хопфа в $H$ и $H / I$ как алгебра Хопфа изоморфна групповой алгебре $k \Omega$. Существует такой эпиморфизм групп $\tau: \mathbb{Z}^{n} \rightarrow \Omega$, что $\Lambda^{H}=\Lambda^{\Omega}$ как векторное пространство порождается всеми такими одночленами $X^{l}$, в которых $l \in(\mathbb{N} \cup 0)^{n} \cap \operatorname{ker} \tau$. Если $\pi: H \rightarrow H / L=k \Omega$ - естественный әпиморфизм, $m o \pi(G(H))=\Omega$.

\section{СПИСОК ЛИТЕРАТУРЫ}

[1] Artamonov V.A., Wisbauer R. Homological properties of quantum polynomials // Algebras and Representation Theory (to appear). [2] Alev J., Chamarie M. // Comm. Algebra. 1992. V. 20. №6. P. 1787-1802. [3] Montgomery S. Hopf Algebras and Their Actions on Rings. Providence RI: Amer. Math. Soc., 1993.

Московский государственный университет им. М. В. Ломоносова
Принято редколлегией 03.10 .2000 